\title{
Investigation of the Genetic Association between Quantitative Measures of Psychosis and Schizophrenia: A Polygenic Risk Score Analysis
}

\author{
Eske M. Derks ${ }^{1,2 * 9}$, Jacob A. S. Vorstman ${ }^{19}$, Stephan Ripke ${ }^{3}$, Rene S. Kahn ${ }^{1}$, The Schizophrenia \\ Psychiatric Genomic Consortium , Roel A. Ophoff ${ }^{1,4,5}$
}

1 Rudolf Magnus Institute of Neuroscience, Department of Psychiatry, University Medical Center Utrecht, The Netherlands, 2 Department of Psychiatry, Academic Medical Center, University of Amsterdam, Amsterdam, The Netherlands, 3 Center for Human Genetic Research, Massachusetts General Hospital, Boston, Massachusetts, United States of America, $\mathbf{4}$ UCLA Center for Neurobehavioral Genetics, Semel Institute for Neuroscience and Human Behavior, Los Angeles, California, United States of America, 5 Department of Medical Genetics, University Medical Center Utrecht, Utrecht, The Netherlands

\begin{abstract}
The presence of subclinical levels of psychosis in the general population may imply that schizophrenia is the extreme expression of more or less continuously distributed traits in the population. In a previous study, we identified five quantitative measures of schizophrenia (positive, negative, disorganisation, mania, and depression scores). The aim of this study is to examine the association between a direct measure of genetic risk of schizophrenia and the five quantitative measures of psychosis. Estimates of the log of the odds ratios of case/control allelic association tests were obtained from the Psychiatric GWAS Consortium (PGC) (minus our sample) which included genome-wide genotype data of 8,690 schizophrenia cases and 11,831 controls. These data were used to calculate genetic risk scores in 314 schizophrenia cases and 148 controls from the Netherlands for whom genotype data and quantitative symptom scores were available. The genetic risk score of schizophrenia was significantly associated with case-control status $(p<0.0001)$. In the case-control sample, the five psychosis dimensions were found to be significantly associated with genetic risk scores; the correlations ranged between.15 and.27 (all $\mathrm{p}<.001$ ). However, these correlations were not significant in schizophrenia cases or controls separately. While this study confirms the presence of a genetic risk for schizophrenia as categorical diagnostic trait, we did not find evidence for the genetic risk underlying quantitative schizophrenia symptom dimensions. This does not necessarily imply that a genetic basis is nonexistent, but does suggest that it is distinct from the polygenic risk score for schizophrenia.
\end{abstract}

Citation: Derks EM, Vorstman JAS, Ripke S, Kahn RS, The Schizophrenia Psychiatric Genomic Consortium, et al. (2012) Investigation of the Genetic Association between Quantitative Measures of Psychosis and Schizophrenia: A Polygenic Risk Score Analysis. PLoS ONE 7(6): e37852. doi:10.1371/journal.pone.0037852

Editor: James Bennett Potash, University of lowa Hospitals \& Clinics, United States of America

Received December 23, 2011; Accepted April 29, 2012; Published June 22, 2012

Copyright: (c) 2012 Derks et al. This is an open-access article distributed under the terms of the Creative Commons Attribution License, which permits unrestricted use, distribution, and reproduction in any medium, provided the original author and source are credited.

Funding: Over 40 United States National Institutes of Health grants and similar numbers of government grants from other countries, along with substantia private and foundation support, enabled this work. We greatly appreciate the sustained efforts of T. Lehner (National Institute of Mental Health) on behalf of the Schizophrenia Psychiatric Genome-Wide Association Study (GWAS) Consortium (PGC). Dr. E.M. Derks is supported by the Netherlands Scientific Organization (NWO; project number 451-080-010). Statistical analyses were carried out on the Genetic Cluster Computer (http://www.geneticcluster.org) which is financially supported by the Netherlands Scientific Organization (NWO 480-05-003). The funders had no role in study design, data collection and analysis, decision to publish, or preparation of the manuscript.

Competing Interests: The authors declare the following interests: Eli Lilly funded portions of the genotyping for CATIE and TOP. P.F.S. received research funding from Eli Lilly in connection with CATIE. T.S.S. received research funding from Eli Lilly and consulting fees from Janssen Pharmaceutica, GlaxoSmithKline and Bristol-Myers Squibb. J.A.L. received research funding from AstraZeneca Pharmaceuticals, Bristol-Myers Squibb, GlaxoSmithKline, Janssen Pharmaceutica and Pfizer and consulting and educational fees from AstraZeneca Pharmaceuticals, Bristol-Myers Squibb, Eli Lilly, Forest Pharmaceuticals, GlaxoSmithKline, Janssen Pharmaceutica, Novartis, Pfizer and Solvay. D.St.C. received research funding from GlaxoSmithKline and Generation Scotland, Genetics Health Initiative. F.A. received funds from Pfizer, Organon and the Foundation for the National Institutes of Health. D.W.B. has received research support from Shire and Forest, has been on the speakers' bureau for Pfizer and has received consulting honoraria from Forest and Jazz. T.W. has received consulting and lecture fees from $H$. Lundbeck A/S. O.A.A. has received Speaker's honorarium from AstraZeneca, Janssen, Bristol-Myers Squibb and GlaxoSmithKline. I.M. has received a Speaker's honorarium from Janssen and AstraZeneca. A.K.M. has received consulting fees or honoraria from Eli Lilly \& Company, Janssen Pharmaceutica, Merck, BristolMeyers Squibb, Pfizer, PGxHealth (a division of Clinical Data, Inc.), Roche Diagnostics and Vanda Pharmaceuticals and has received research support from Eli Lilly \& Company. T.L. has received consulting fees or honoraria from Merck, Eli Lilly \& Company, Golden Helix, Inc., InforMed Insights and PGxHealth (a division of Clinical Data, Inc.). I.B. has been an advisory board member, consultant and lecturer for AstraZeneca, Bristol-Myers Squibb, Eli Lilly, EGIS, Janssen, H. Lundbeck A/S, Novartis, Pfizer, Richter and Schering-Plough and received a grant for an investigator-initiated study from H. Lundbeck A/S. J.J.M. has received consulting and speaker's fees from Johnson \& Johnson, Schering-Plough and Eli Lilly. C.P. has received grant support from Janssen-Cilag, Eli Lilly, Hospira (Mayne) and AstraZeneca, provided consultancy to Janssen-Cilag, Eli Lilly, Hospira (Mayne), AstraZeneca, Pfizer and Schering-Plough and has undertaken investigator-initiated studies supported by Eli Lilly, Hospira, Janssen Cilag and AstraZeneca. The Denmark-Aarhus group (The GEMS Stud with principal investigators A.D.B., O.M. and P.B.M.) received research funding from $\mathrm{H}$. Lundbeck A/S. E.G.J. has served as an unpaid consultant for Eli Lilly. H.S., S.S., and K.S. are employed at deCODE Genetics, Reykjavik, Iceland. MH is employed at Illumina, Inc., La Jolla, California, USA. PM is employed at NeuroSearch A/S, Ballerup, Denmark. TFO is employed at ARoS Applied Biotechnology A/S, Skejby, Denmark. There are no patents, products in development or marketed products to declare. This does not alter the authors' adherence to all the PLOS ONE policies on sharing data and materials, as detailed online in the guide for authors.

- A full list of the authors in the Schizophrenia Psychiatric Genomic Consortium is provided in the Acknowledgements

*E-mail: e.m.derks@amc.uva.nl

9 These authors contributed equally to this work. 


\section{Introduction}

In clinical practice as well as in most research schizophrenia is conceptualized as a categorical entity, allowing for a distinction in the population between affected and unaffected. Yet, it has been proposed that psychotic symptoms, in essence the same as those observed in individuals with schizophrenia, can also be measured at subclinical levels in individuals without schizophrenia in the general population [1]. The concept of a psychosis continuum implies that schizophrenia is not a categorical disorder, but rather the extreme expression of otherwise more or less continuously distributed traits in the population [2]. This view has gained momentum in recent years. For instance the results of a metaanalysis showed prevalence rates of psychotic experiences and symptoms of approximately $4-8 \%$ in the general population. In addition, some of the previously identified risk factors for schizophrenia, including cannabis, traumatic experiences and urbanicity, also increase the risk of psychotic experiences in the non-clinical population [3]. A recent study demonstrated that, using affectedness of relatives as a proxy, a higher genetic loading increased the risk of psychotic symptoms in subjects without a clinical psychotic disorder [4]. To date, no study has explored a possible correlation between a direct continuous measure of genetic risk and the continuous psychosis phenotype. The question is not trivial; finding such correlation would provide strong genetic evidence for the concept of the psychosis continuum. Inversely, the demonstration of such correlation would provide a strong argument for investigating the dimensional scale of psychotic experiences or symptoms in genetic studies [5].

Reporting on data from the International Schizophrenia Consortium, Purcell and colleagues presented evidence in support of a polygenic contribution to schizophrenia [6]. They demonstrated that the available Genome Wide Association (GWA) findings are compatible with a large number of shared loci each with very small odds ratios contributing to disease susceptibility. Based on the nominally associated alleles in a discovery sample, a quantitative polygenic risk score was calculated. Subsequently, when comparing this polygenic risk score between cases and controls in two independent schizophrenia samples a significantly higher signal was detected in cases. As was proposed recently by Plomin and colleagues, the involvement of multiple genes indicated by the GWAS results for a disorder suggests that the genetic liability may be distributed quantitatively rather than qualitatively. This in turn raised the question to which extent this distribution of polygenic liability is mirrored in a similar distribution of quantitative traits which compose the disorder [7].

Interestingly, the schizophrenia-derived polygenic risk score was also shown to be significantly increased in bipolar disorder [6]. This could be interpreted as an indication that genetic liability can be present with regard to certain symptom domains, rather than for a specific (categorically defined) syndrome of symptoms. In other words, it can be argued that the liability for schizophrenia is composed of co-occurring different genetic liabilities for different symptom domains. This was supported by recent findings suggesting the presence of two distinct polygenic risk scores: one that relates to expression of a 'bipolar disorder-like' phenotype and one that is associated with expression of 'schizophrenia-like' psychotic symptoms [8].

Several studies have investigated whether genetic factors contribute to symptom dimensions of schizophrenia. Results of twin studies suggest heritability of symptoms of disorganization [9] and reality distortion, i.e. hallucinations and/or delusions [10]. A meta-analysis of the results of studies on symptom concordance in schizophrenia affected sibling pairs demonstrated significant correlations within siblings for the dimensions psychomotor poverty, reality distortion and disorganization, with the latter showing the highest correlation coefficient [11]. Individual genes have also been suggested to differentially impact the different quantitative symptom dimensions (reviewed in $[12,13]$ ).

We recently performed factor analyses on 79 symptoms related to schizophrenia in a large sample of over four thousand subjects, approximately half of which were healthy controls, while the other half of the subjects were diagnosed with affective or non affective psychotic disorders or non psychotic mood disorders [14]. This was a first attempt to detect latent dimensions for schizophrenia in a sample that included both psychotic and non-psychotic patients as well as healthy controls. Our analyses indicated five continuous dimensions of schizophrenia: positive, negative, disorganisation, mania, and depression. Importantly, these results have provided us with measures that express five phenotypic components of the schizophrenia phenotype as continuous, quantitative traits.

The current study was set up to examine the correlation between a direct measure of genetic risk of schizophrenia, using the polygenic risk score, and quantitative measures of schizophrenia symptoms, using the five continuous symptom dimensions of schizophrenia derived from our previous study. We hypothesize a positive correlation between the polygenic risk score and one or more of the symptom dimension scales in individuals with and without a diagnosis of schizophrenia.

We propose that if a significant correlation can be confirmed, this finding would provide genetic evidence for the psychosis continuum concept by substantiating the notion proposed by Plomin et al., namely that the polygenic liability is mirrored in a similar distribution of quantitative traits which compose the disorder [7].

\section{Methods}

\section{Subjects}

From a sample of 715 schizophrenia cases and 643 controls from The Netherlands, genotypic data of 704 cases and 631 controls passed Quality Control (QC) criteria. We additionally removed 10 subjects ( 5 cases and 5 controls) who were indicated as outliers according to a principal component analysis performed in EIGENSOFT [15]. This resulted in the final case-control sample including data of 699 cases and 626 controls. Detailed phenotypic assessments (i.e., the Comprehensive Assessment of Symptoms and History (CASH) [16]) was collected in a smaller subsample including 314 schizophrenia patients and 148 controls. This is the sample that was used for the analyses of the symptom dimension scores. The controls had no history of psychiatric disorder. All patients and controls had at least three grandparents of Dutch ancestry. The study was approved by the institutional ethical committee and informed consent was obtained from all participants. A more detailed description of the inclusion protocol has been described elsewhere [17].

\section{Genotyping and Quality Control Procedure}

All genome-wide genotyping for the GWAS was performed on Human- Hap550v3 BeadArrays using the Infinium II assay (Illumina) at the Southern California Genotyping Consortium (SCGC) at UCLA, Los Angeles, USA.

An extensive quality control $(\mathrm{QC})$ protocol was carried out, the procedure has been described in full detail [18]. Briefly, SNPs were included if the missing rate was $<.02$, the SNP frequency difference to HapMap was $<.15$, the difference missing rate per SNP between cases and controls <.02), and Hardy-Weinberg Equilibrium was not violated in controls $\left(\mathrm{p}<10^{-6}\right)$. Individuals 
were included if the missing rate was $<.02$. We removed one member of a pair of observations in case of duplication or cryptic relatedness. Finally, ten subjects were indicated as outliers according to the principal component analysis and were removed from subsequent analyses. The QC protocol resulted in a sample of 699 cases and 626 controls. The genomic inflation factor of this sample was 1.02; the QQ plot is shown in Figure S1.

\section{Statistical Analysis}

We have previously calculated liability scores on the five psychosis dimensions (i.e., disorganization, positive, negative, mania, and depression) in a confirmatory factor analysis including CASH lifetime rated symptoms from 4,286 subjects. Of these individuals, $\mathrm{N}=1,965$ were healthy controls while the remaining individuals were diagnosed with a psychiatric disorder $(\mathrm{N}=1,085$ schizophrenia or schizophreniform disorder; $\mathrm{N}=160$ schizoaffective disorder; $\mathrm{N}=202$ bipolar disorder; $\mathrm{N}=480$ major depression; $\mathrm{N}=388$ other psychiatric diagnoses) [14]. The subjects included in the present study are a subset of this larger sample.

Estimates of the log of the odds ratios of case/control allelic association tests were obtained from the Psychiatric GWAS Consortium (PGG) sample (but excluding the Utrecht/UCLA sample) which included Single Nucleotide Polymorphism (SNP) data $(\mathrm{N}=1,241,601)$ from 8,690 schizophrenia cases and 11,831 controls. SNPs were imputed with HapMap-3 [19] as the reference panel; confidence metrics in the single datasets were set at 0.1 .

We selected SNPs which were associated with case-control status below a fixed p-value. Three selection thresholds were applied; all SNPs associated at $\mathrm{p}<.5$ (selection 1); all SNPs associated at $\mathrm{p}<.1$ (selection 2); and all SNPs associated at $\mathrm{p}<.01$ (selection 3). LD pruning was applied to select SNPs which are in approximate linkage equilibrium with each other. We used the indep option in PLINK with the default values for the parameters (i.e., window size of $50 \mathrm{~kb}$, the number of SNPs to shift the window at each step $=5$, and a Variance Inflation Factor $(\mathrm{VIF})$ threshold of 2 [20]. The total number of SNPs in the three analyses was 63,935 (selection $1 ; \mathrm{p}<.50$ ); 14,654 (selection 2; $\mathrm{p}<.10) ; 1,954$ (selection $3 ; \mathrm{p}<.01$ ).

Genetic risk scores were calculated in PLINK [20] using the method described by Purcell and colleagues [6].

Briefly, risk scores were calculated based on an individual's genotype. For each SNP, the log of the odds ratio of an allele was multiplied by $(0,1$, or 2$)$ depending on the number of risk alleles that an individual carries. The total polygenic risk score is simply a sum across SNPs.

In the subsequent analyses, we aimed to correct for the possible presence of population stratification, by adjusting for the first 10 principal components which were calculated with EIGENSOFT [15]. A logistic regression analysis was used to investigate whether the genetic risk score is indeed significantly associated with casecontrol status in our independent sample to replicate the results of Purcell et al. [6]. Nagelkerke R squared was used to compare the percentage of variance in case-control status explained by the first 10 principal components with the percentage of variance explained by the first 10 principal components and the genetic risk score.

Next, we calculated partial correlations between genetic risk scores (including all SNPs associated at $\mathrm{p}<.5$ ) and dimension scores, adjusting for the first 10 principal components in a sample of 314 schizophrenia cases and 148 controls. This analysis was performed in the total sample and in cases and controls separately. Power analyses performed in statistical package $\mathrm{R}$ [21] demonstrated that within cases and controls this study had $80 \%$ power to detect correlations of.22 and.16 respectively, using a type-I error rate of $5 \%$. We also repeated the case-control analysis in this smaller subsample to facilitate comparison of the results of the case-control analysis and the dimension score analyses.

\section{Results}

A graphical representation of the distribution of the genetic risk scores is provided in Figure S2 for cases and controls respectively.

The polygenic risk scores were standardized (i.e., mean $=0$ and $\mathrm{SD}=1$ ) in the total sample. A summary of mean genetic risk scores by case/control status is provided in Table 1 for each of the three selections. The logistic regression analysis indicated that the 10 principal components explained $1.5 \%$ of the variance in case/control status $\left(\mathrm{X}^{2}(10)=15.45, \mathrm{p}=.12\right)$. The prediction of case-control status significantly improved by including the genetic risk factor as a predictor with the proportion of variance increasing to $2.9 \%, 4.7 \%$, and $5.1 \%$ based on the inclusion of SNPs associated at $\mathrm{p}<.01,0.10$, and 0.50 , respectively. Substracting the variance explained by the principal components, this implies that $\sim 1.4$ to $3.6 \%$ of the variance in case-control status is explained by the genetic risk score. The association between genetic risk score and casecontrol status was highly significant. For example, using a threshold of $\mathrm{p}<0.50$, the regression coefficient of the genetic risk score was 0.36 (Wald $=5.89, \mathrm{p}<0.0001$ ). Including all SNPs associated at $\mathrm{p}<0.50$, the mean genetic risk scores were.13 $(\mathrm{SD}=.98)$ and $-.18(\mathrm{SD}=.94)$ in cases and controls, respectively (see Figure S2). Exclusion of the SNPs in the extended MHC region (6p21.31-6p22.1) did not affect the results.

Next, we studied the association between psychosis dimensions and genetic risk scores (see Table 1). In the total sample of cases and controls, the correlations between psychosis dimen-

Table 1. Association of genetic risk scores with symptom dimensions and case-control status across thresholds.

\begin{tabular}{|c|c|c|c|c|c|c|}
\hline & \multicolumn{5}{|c|}{$\begin{array}{l}\text { Pearson correlations between genetic risk scores and symptom dimensions } \\
\text { in the total sample and by status }\end{array}$} & \multirow{2}{*}{$\begin{array}{l}\text { Case-control } \\
\text { status }\end{array}$} \\
\hline & $\begin{array}{l}\text { Positive Total } \\
\text { (case/control) }\end{array}$ & $\begin{array}{l}\text { Negative Total } \\
\text { (case/control) }\end{array}$ & $\begin{array}{l}\text { Disorganisation Total } \\
\text { (case/control) }\end{array}$ & $\begin{array}{l}\text { Mania Total } \\
\text { (case/control) }\end{array}$ & $\begin{array}{l}\text { Depression Total } \\
\text { (case/control) }\end{array}$ & \\
\hline Genetic risk score: $\mathrm{p}<.5$ & $.15^{*}(-.01 / .06)$ & $.19^{* *}(.03 / .07)$ & $.09(-.09 / .05)$ & $.12^{*}(-.05 / .02)$ & $.16^{* *}(-.06 / .06)$ & $.13 /-.18^{* *}$ \\
\hline Genetic risk score: $p<.1$ & $.17^{* *}(.03 / .05)$ & $.17^{* *}(.03 / .08)$ & $.08(-.10 / .08)$ & $.12(-.07 / .08)$ & $.15^{*}(-.06 / .08)$ & $.14 /-.17^{* *}$ \\
\hline Genetic risk score: $\mathrm{p}<.01$ & $.13 *(.06 / .03)$ & $.13^{*}(.04 / .02)$ & $.03(-.08 /-.09)$ & $.07(.01 /-.06)$ & $.11(.03 /-.08)$ & $.09 /-.12^{* *}$ \\
\hline
\end{tabular}


A

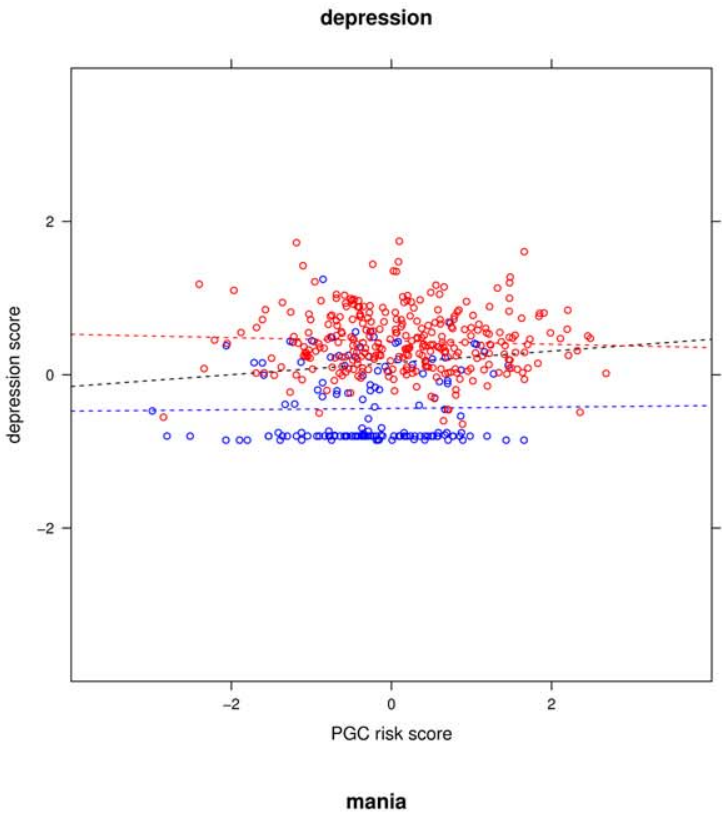

C

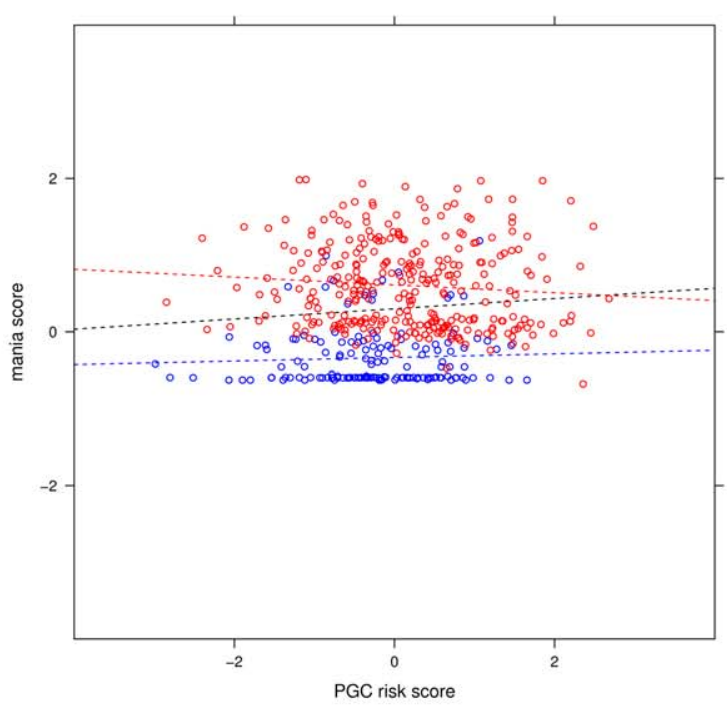

E

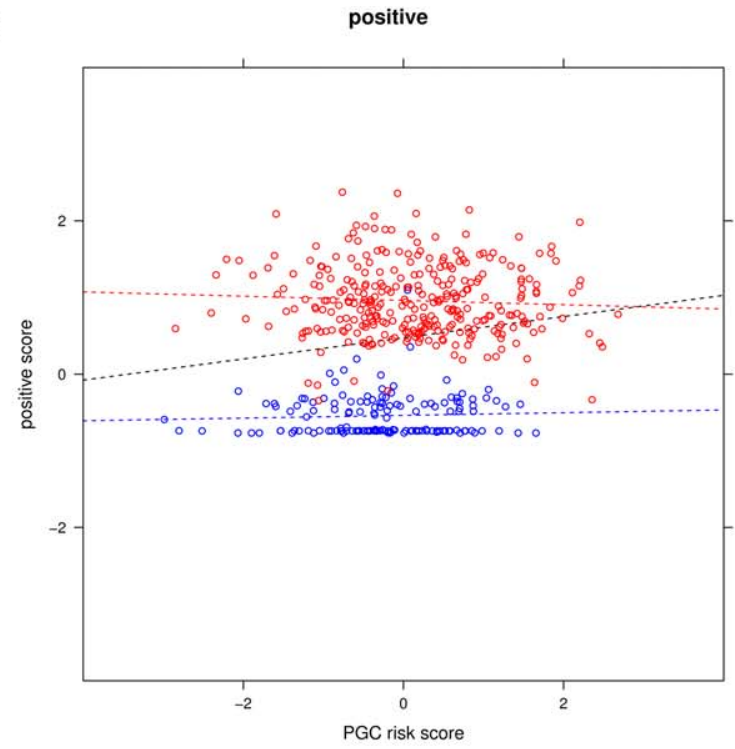

B

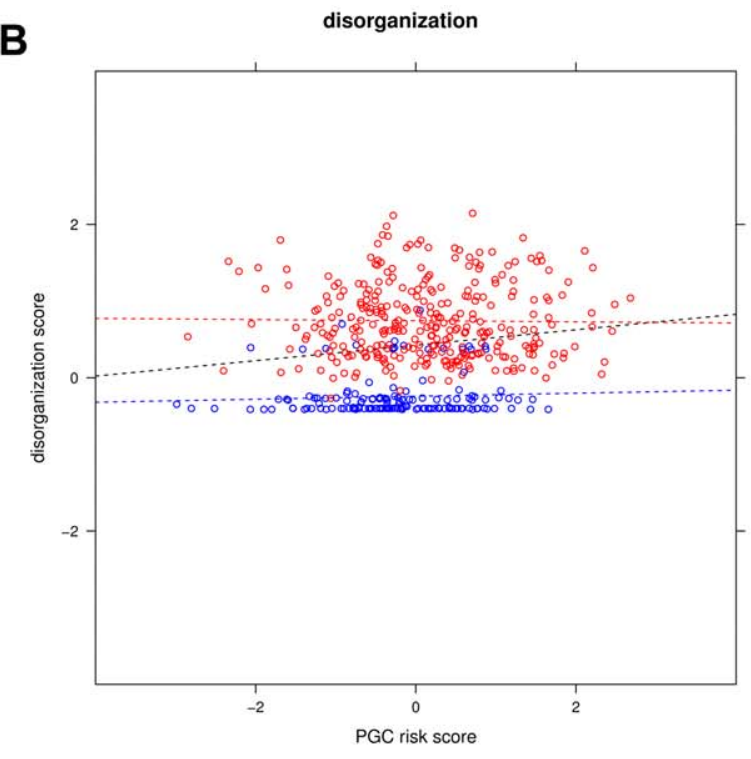

D

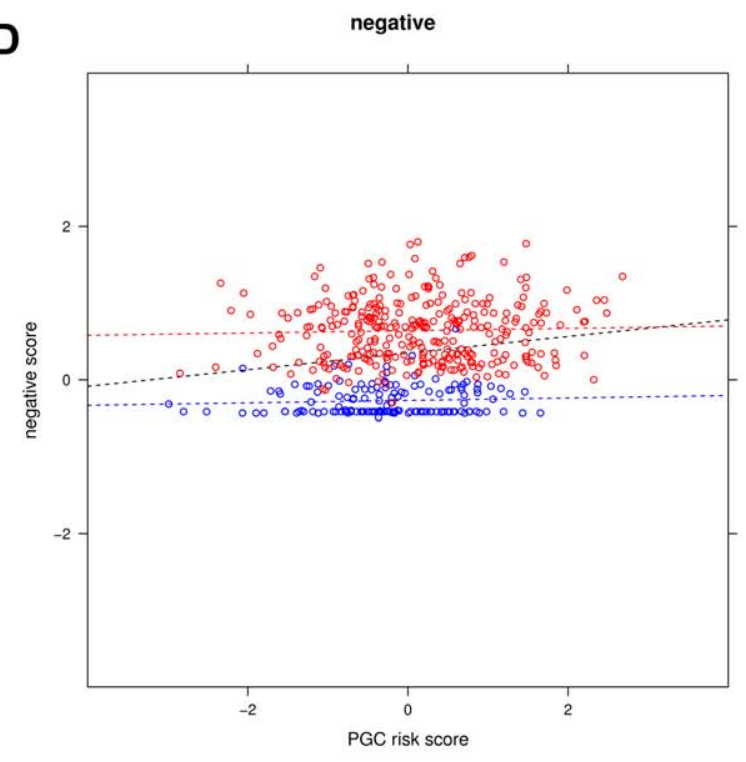


Figure 1a-e. Associations between symptom dimension scores and genetic risk scores. Legend "The red dots represent cases and the blue dots represent controls. The regression lines represent the association between genetic risk score and dimension scores in the total sample (black) in cases (red) and in controls (blue).

doi:10.1371/journal.pone.0037852.g001

sions and genetic risk scores range between.09 and.19. These correlations were statistically significant $(\mathrm{p}<.01)$ for the dimensions positive $(\mathrm{r}=.15)$, negative $(\mathrm{r}=.19)$, mania $(\mathrm{r}=.12)$, and depression $(r=.16)$ while the correlation of the dimensions disorganization $(r=.09)$ was not statistically significant $(\mathrm{p}=.04)$. The positive correlations are not unexpected since case-control status is associated both with dimension scores and genetic risk scores. Therefore, we continued our analyses by testing whether genetic risk scores are significantly associated with psychosis dimension scores in the cases and controls separately. In schizophrenia cases, the correlations ranged between -.06 and.04 and were not significantly different from zero (all $\mathrm{p}>$.10). Similarly, the correlations in controls were not significant; the range was between.02 and.07 (all p<.10).

Figure la-e show the associations between genetic risk scores and psychosis dimensions in the total sample with cases and controls plotted in different colors. As dimension scores were assessed in a relatively small subsample $(24 \%$ of the controls and $45 \%$ of the cases), we repeated the case-control analysis in this subsample to facilitate comparison of the results. Including all SNPs associated at $\mathrm{p}<.50,7 \%$ of the variance was explained by the 10 principal components $\left(\mathrm{X}^{2}(10)=22.66, \mathrm{p}=.01\right)$ which increased to $13 \%$ after inclusion of the genetic risk score $\left(\mathrm{X}^{2}(11)=43.23, \mathrm{p}<.0001\right)$.

\section{Discussion}

The aim of this study was to investigate the correlation between the polygenic liability for schizophrenia and quantitative domains of schizophrenia symptoms in schizophrenia cases and healthy controls. In the current sample, we replicate the findings by Purcell et al. [6] and show that the polygenic risk score effectively predicts schizophrenia status in our sample. Given that both the dimension scores and the genetic scores are highly associated with case-control status it is not surprising that the polygenic score was also significantly correlated with each of the five schizophrenia dimensions when analyzing the entire sample. The polygenic risk score did not have a significant correlation with any of the five symptoms dimensions when cases and controls were analyzed separately. Therefore, we conclude that the genetic basis of severity differences within diagnostic subgroups (i.e., cases vs. controls) is not shared with the genetic basis of case-control status.

There are several possible explanations for this finding. First, in reality such genetic basis exists but the score alleles (SNPs) used in the current study do not index severity of the schizophrenia dimensions selected in the current study. SNPs were selected based on their association with case-control status and possibly, other score alleles may be correlated with the schizophrenia dimensions used in the current study. It is also possible that the used score alleles may be correlated with schizophrenia dimensions other than those used in our study. Alternatively, the explained variance is very small with correlations $<.2$, which are potentially not detected due to a lack of statistical power given the sample size of this study. A whole different explanation may be that while there is a continuously distributed genetic liability correlated with schizophrenia dimensions, this genetic liability is not based on common allelic (SNP) variants. For instance, a continuously distributed measure based on rare genetic variants or on epigenetic variation is possible in theory. The most dramatic explanation for our observation that the schizophrenia polygenic risk score does not predict severity of symptom dimensions could be the absence of a continuously distributed genetic liability that explains the observed psychosis continuum. Please note that this is not inconsistent with the available evidence indicating a genetic contribution to schizophrenia dimensions; results so far [11] do not provide evidence that such genetic contribution is present when adjusting for case-control status.

It should also be noted that, based on the results of our previous study [14], we have chosen for the inclusion of five symptom dimensions. The results of factor analyses largely depend on the content of the items that are included. If we would have included items of additional instruments, the number and interpretation of the resulting factors could have been different. The results of factor analytical studies have been discussed by Peralta and Cuesta [22] who showed that the number of factors ranged from 4 to 11 , depending on the content of the items included in the analyses. The inclusion of additional factors (e.g., psychomotor poverty) could result in different findings and we hope that other research groups, who have used different instruments for the assessment of psychosis will address this question. Future collaborative studies should aim to further elucidate the genetic basis of quantitative symptom dimensions by combining symptom ratings assessed in psychiatric cases (e.g., schizophrenia, bipolar disorder, depression), and healthy controls.

\section{Supporting Information}

Figure S1 QQ plot of the UGLA case-control sample. This figure plots the expected $-\log _{10}(\mathrm{p})$ at the $\mathrm{x}$-axis and the observed $-\log _{10}(\mathrm{p})$ at the $\mathrm{y}$-axis.

(DOC)

Figure S2 Distribution of genetic risk scores in schizophrenia cases and controls. This figure shows the distribution of the genetic risk score in cases and controls.

(DOC)

\section{Acknowledgments}

The Schizophrenia Psychiatric Genomic Gonsortium

Gardiff UK: Michael C. O'Donovan (Cardiff University, Cardiff, UK), Nicholas Craddock (Cardiff University, Cardiff, UK), Peter A. Holmans (Cardiff University, Cardiff, UK), Marian Hamshere (Cardiff University, Cardiff, UK), Hywel J. Williams (Cardiff University, Cardiff, UK), Valentina Moskvina (Cardiff University, Cardiff, UK), Sarah Dwyer (Cardiff University, Cardiff, UK), Lyudmila Georgieva (Cardiff University, Cardiff, UK), Stan Zammit (Cardiff University, Cardiff, UK), Michael J. Owen (Cardiff University, Cardiff, UK).

CATIE: Patrick F. Sullivan (Karolinska Institutet, Stockholm, Sweden, and University of North Carolina, Chapel Hill, USA), Dan-Yu Lin (University of North Carolina, Chapel Hill, USA), Edwin van den Oord (Virginia Commonwealth University, Richmond, USA), Yunjung Kim (University of North Carolina at Chapel Hill, Chapel Hill, USA), T. Scott Stroup (Columbia University, New York, USA), Jeffrey A Lieberman (Columbia University, New York, USA).

ISC - Aberdeen: David St Clair (University of Aberdeen, Aberdeen, Scotland).

ISC - Cardiff: George K. Kirov (Cardiff University, Cardiff, UK), Michael C. O'Donovan (Cardiff University, Cardiff, UK), Peter A. Holmans (Cardiff University, Cardiff, UK), Lyudmila Georgieva (Cardiff University, Cardiff, UK), Ivan Nikolov (Cardiff University, Cardiff, UK), 
Hywel J. Williams (Cardiff University, Cardiff, UK), Draga Toncheva (University Hospital Maichin Dom, Sofia, Bulgaria), Vihra Milanova (Alexander University Hospital, Sofia, Bulgaria), Michael J. Owen (Cardiff University, Cardiff, UK).

ISG - Dublin: Derek W. Morris (Trinity College Dublin, Dublin, Ireland), Colm T. O'Dushlaine (Trinity College Dublin, Dublin, Ireland), Elaine Kenny (Trinity College Dublin, Dublin, Ireland), Emma M. Quinn (Trinity College Dublin, Dublin, Ireland), Michael Gill (Trinity College Dublin, Dublin, Ireland), Aiden Corvin (Trinity College Dublin, Dublin, Ireland).

ISG - Edinburgh: Douglas H. R. Blackwood (University of Edinburgh, Edinburgh, UK), Kevin A. McGhee (University of Edinburgh, Edinburgh, UK), Ben Pickard (University of Strathclyde, Glasgow, UK), Pat Malloy (University of Edinburgh, Edinburgh, UK), Alan W. Maclean (University of Edinburgh, Edinburgh, UK), Andrew McIntosh (University of Edinburgh, Edinburgh, UK).

ISG - London: Andrew McQuillin (University College London Medical School, London, UK), Khalid Choudhury (University College London Medical School, London, UK), Susmita Datta (University College London Medical School, London, UK), Jonathan Pimm (University College London Medical School, London, UK), Srinivasa Thirumalai (West Berkshire NHS Trust, Reading, UK), Vinay Puri (University College London Medical School, London, UK), Robert Krasucki (University College London Medical School, London, UK), Jacob Lawrence (University College London Medical School, London, UK), Digby Quested (University of Oxford, Oxford, UK), Nicholas Bass (University College London Medical School, London, UK), Hugh Gurling (University College London Medical School, London, UK)

ISC - Portugal: Michele T. Pato (University of Southern California, Los Angeles, USA), Carlos N. Pato (University of Southern California, Los Angeles, USA), Ayman Fanous (Washington VA Medical Center, Washington, DC, USA, Georgetown University School of Medicine, Washington, DC, USA, and Virginia Commonwealth University School of Medicine, Richmond, USA)

ISG - SW1, ISC - SW2: Christina M. Hultman (Karolinska Institutet, Stockholm, Sweden), Paul Lichtenstein (Karolinska Institutet, Stockholm, Sweden), Sarah E. Bergen (Massachusetts General Hospital, Boston, USA), Shaun Purcell (Broad Institute, Cambridge, USA), Edward Scolnick (Broad Institute, Cambridge, USA), Pamela Sklar (Massachusetts General Hospital, Boston, USA, and Mount Sinai School of Medicine, New York, USA), Patrick F. Sullivan (Karolinska Institutet, Stockholm, Sweden, and University of North Carolina, Chapel Hill, USA).

MGS: Pablo V. Gejman (North Shore University Health System, Evanston, USA, and University of Chicago, Chicago, USA), Alan R. Sanders (NorthShore University HealthSystem, Evanston, USA, and University of Chicago, Chicago, USA), Jubao Duan (North Shore University Health System, Evanston, USA, and University of Chicago, Chicago, USA), Douglas F. Levinson (Stanford University, Stanford, USA), Jianxin Shi (National Cancer Institute, Bethesda, USA), Nancy G. Buccola (Louisiana State University, New Orleans, USA), Bryan J. Mowry (Queensland Brain Institute, Brisbane, Australia), Robert Freedman (University of Colorado Denver, Aurora, USA), Farooq Amin (Emory University, Atlanta, USA, and Atlanta Veterans Affairs Medical Center, Atlanta, USA), DW Black Donald W. Black (University of Iowa, Iowa City, USA), Jeremy M. Silverman (Mount Sinai School of Medicine, New York, USA, and Veterans Affairs Medical Center, New York, USA), William F. Byerley (University of California at San Francisco, San Francisco, USA, and Northern California Institute for Research And Education, San Francisco, USA), C. Robert Cloninger (Washington University, St. Louis, USA).

SGENE - Bonn: Sven Cichon (University of Bonn, Bonn, Germany, and Research Center Juelich, Juelich, Germany), Marcella Rietschel (University of Bonn, Bonn, Germany, and University of Heidelberg, Mannheim, Germany), Markus M. Nöthen (University of Bonn, Bonn, Germany, and Research Center Juelich, Juelich, Germany), Wolfgang Maier (University of Bonn, Bonn, Germany), Thomas G. Schulze (University of Göttingen), Manuel Mattheisen (Brigham and Women's Hospital and Harvard Medical School, Boston, USA, and University of Bonn, Bonn, Germany).

SGENE - Copenhagen: Thomas Hansen (Institute of Biological Psychiatry, MHC. Sct. Hans, University of Copenhagen), Line Olsen (Institute of Biological Psychiatry, MHC. Sct. Hans, University of Copenhagen), Andrés Ingason (Institute of Biological Psychiatry, MHC.
Sct. Hans, University of Copenhagen), Klaus D. Jakobsen (Institute of Biological Psychiatry, MHC. Sct. Hans, University of Copenhagen), Henriette Schmock (Institute of Biological Psychiatry, MHC. Sct. Hans, University of Copenhagen), Celina Skjødt (Institute of Biological Psychiatry, MHC. Sct. Hans, University of Copenhagen), Anders Rosengren (Institute of Biological Psychiatry, MHC. Sct. Hans, University of Copenhagen), Louise KE Høffding (Institute of Biological Psychiatry, MHC. Sct. Hans, University of Copenhagen), Johan H Thygersen (Institute of Biological Psychiatry, MHC. Sct. Hans, University of Copenhagen), Anders Vangkilde (Institute of Biological Psychiatry, MHC. Sct. Hans, University of Copenhagen), Thomas Werge (Institute of Biological Psychiatry, MHC. Sct. Hans, University of Copenhagen).

SGENE - Munich: Ina Giegling (Ludwig-Maximilians University, Munich, Germany), Annette M. Hartmann (Ludwig-Maximilians University, Munich, Germany), Heike Konnerth (Ludwig-Maximilians University, Munich, Germany), Marion Friedl (Ludwig Maximilians University, Munich, Germany), Bettina Konte (Ludwig-Maximilians University, Munich, Germany), Pierandrea Muglia (University of Toronto, Toronto, Canada. NeuroSearch A/S, Ballerup, Denmark), Dan Rujescu (LudwigMaximilians University, Munich, Germany).

SGENE - TOP3: Srdjan Djurovic (University of Oslo, Oslo, Norway, and Oslo University Hospital, Oslo, Norway), Morten Mattingsdal (University of Oslo, Oslo, Norway, and Sørlandet Hospital, Kristiansand, Norway), Ingrid Agartz (University of Oslo, Oslo, Norway, and Diakonhjemmet Hospital, Oslo, Norway), Ingrid Melle (University of Oslo, Oslo, Norway, and Oslo University Hospital, Oslo, Norway), Ole A. Andreassen (University of Oslo, Oslo, Norway, and Oslo University Hospital, Oslo, Norway).

SGENE - UCLA: Roel A. Ophoff (University Medical Center Utrecht, Utrecht, The Netherlands, and University of California at Los Angeles, Los Angeles, USA), Rita M. Cantor (University of California at Los Angeles, Los Angeles, USA), Nelson B. Freimer (University of California at Los Angeles, Los Angeles, USA), René S. Kahn (University Medical Center Utrecht, Utrecht, The Netherlands), Don H. Linszen (University of Amsterdam, Amsterdam, The Netherlands), Jim van Os (Maastricht University Medical Centre, Maastricht, The Netherlands), Durk Wiersma (University of Groningen, Groningen, The Netherlands), Richard Bruggeman (University of Groningen, Groningen, The Netherlands), Wiepke Cahn (University Medical Center Utrecht, Utrecht, The Netherlands), Lieuwe de Haan (Academic Medical Centre University of Amsterdam, Amsterdam, The Netherlands), Lydia Krabbendam (Maastricht University Medical Centre, Maastricht, The Netherlands), Inez Myin-Germeys (Maastricht University Medical Centre, Maastricht, The Netherlands), Eric Strengman (University Medical Center Utrecht, Utrecht, The Netherlands)

Zucker Hillside: Anil K. Malhotra (The Zucker Hillside Hospital Division of the North Shore, Glen Oaks, USA, The Feinstein Institute for Medical Research, Manhasset, USA, and Albert Einstein College of Medicine of Yeshiva University, Bronx, New York, USA), Todd Lencz (The Zucker Hillside Hospital Division of the North Shore, Glen Oaks, USA, The Feinstein Institute for Medical Research, Manhasset, USA, and Albert Einstein College of Medicine of Yeshiva University, Bronx, New York, USA).

Statistical Analyses: Stephan Ripke (Massachusetts General Hospital, Boston, Massachusetts, USA), Mark J Daly (Massachusetts General Hospital, Boston, Massachusetts, USA), Peter A. Holmans (Cardiff University, Cardiff, UK), Dan-Yu Lin (University of North Carolina, North Carolina, USA), Shaun Purcell (Broad Institute, Cambridge, Massachusetts, USA), Frank Dudbridge (London School of Hygiene and Tropical Medicine, London, UK), Ben M. Neale (Broad Institute, Cambridge, Massachusetts, USA), Lizzy Rossin (Massachusetts General Hospital, Boston, Massachusetts, USA), Peter M. Visscher (Queensland Institute of Medical Research, Brisbane, Queensland, Australia), Danielle Posthuma (Vrije Universiteit, Amsterdam, The Netherlands), Douglas M. Ruderfer (Massachusetts General Hospital, Boston, Massachusetts, USA).

Phenotype Group: Kenneth S Kendler (Virginia Commonwealth University School of Medicine, Richmond, VA, USA), Ayman Fanous (Washington VA Medical Center, Washington, DC, USA, Georgetown University School of Medicine, Washington, DC, USA, and Virginia Commonwealth University School of Medicine, Richmond, USA), Aiden 
Corvin (Neuropsychiatric Genetics Research Group, Trinity College Dublin, Dublin, Ireland).

PGG-SZ group Chair: Michael G O'Donovan (Gardiff University, Cardiff).

\section{References}

1. van Os J, Kapur S (2009) Schizophrenia. Lancet 374: 635-645.

2. van Os J, Hanssen M, Bijl RV, Ravelli A (2000) Strauss (1969) revisited: a psychosis continuum in the general population? Schizophr Res 45: 11-20.

3. van Os J, Linscott RJ, Myin-Germeys I, Delespaul P, Krabbendam L (2009) A systematic review and meta-analysis of the psychosis continuum: evidence for a psychosis proneness-persistence-impairment model of psychotic disorder. Psychol Med 39: 179-195.

4. Binbay T, Drukker M, Elbi H, Aksu TF, Ozkinay F, et al. (in press) Testing the Psychosis Continuum: Differential Impact of Genetic and Nongenetic Risk Factors and Comorbid Psychopathology Across the Entire Spectrum of Psychosis. Schizophr Bull.

5. Plomin R, Owen MJ, McGuffin P (1994) The genetic basis of complex human behaviors. Science 264: 1733-1739.

6. Purcell SM, Wray NR, Stone JL, Visscher PM, O'Donovan MC, et al. (2009) Common polygenic variation contributes to risk of schizophrenia and bipolar disorder. Nature 460: 748-752.

7. Plomin R, Haworth CM, Davis OS (2009) Common disorders are quantitative traits. Nat Rev Genet 10: 872-878.

8. Hamshere ML, O'Donovan MC, Jones IR, Jones L, Kirov G, et al. (2011) Polygenic dissection of the bipolar phenotype. Br J Psychiatry 198: 284-288.

9. Cardno AG, Sham PC, Murray RM, McGuffin P (2001) Twin study of symptom dimensions in psychoses. Br J Psychiatry 179: 39-45.

10. Torgersen S, Edvardsen J, Oien PA, Onstad S, Skre I, et al. (2002) Schizotypal personality disorder inside and outside the schizophrenic spectrum. Schizophr Res 54: 33-38.

11. Rietkerk T, Boks MP, Sommer IE, Liddle PF, Ophoff RA, et al. (2008) The genetics of symptom dimensions of schizophrenia: review and meta-analysis. Schizophr Res 102: 197-205.

12. Fanous AH, Kendler KS (2005) Genetic heterogeneity, modifier genes, and quantitative phenotypes in psychiatric illness: searching for a framework. Mol Psychiatry 10: 6-13.

\section{Author Contributions}

Conceived and designed the experiments: RSK PGC RAO. Performed the experiments: ED JV SR. Analyzed the data: ED. Wrote the paper: ED JV SR RAO.

13. Fanous AH, Kendler KS (2008) Genetics of clinical features and subtypes of schizophrenia: a review of the recent literature. Curr Psychiatry Rep 10: 164 170 .

14. Derks EM, Allardyce J, Boks MP, Vermunt JK, Hijman R, et al. (in press). Kraepelin Was Right: A Latent Class Analysis of Symptom Dimensions in Patients and Controls. Schizophr Bull.

15. Price AL, Patterson NJ, Plenge RM, Weinblatt ME, Shadick NA, et al. (2006) Principal components analysis corrects for stratification in genome-wide association studies. Nat Genet 38: 904-909.

16. Andreasen NC, Flaum M, Arndt S (1992) The Comprehensive Assessment of Symptoms and History (CASH). An instrument for assessing diagnosis and psychopathology. Arch Gen Psychiatry 49: 615-623.

17. Rietschel M, Mattheisen M, Degenhardt F, Kahn RS, Linszen DH, et al. (2011) Association between genetic variation in a region on chromosome 11 and schizophrenia in large samples from Europe. Mol Psychiatry.

18. Ripke S, Sanders AR, Kendler KS, Levinson DF, Sklar P, et al, (2011) Genomewide association study identifies five new schizophrenia loci. Nat Genet 43: 969976.

19. Altshuler DM, Gibbs RA, Peltonen L, Altshuler DM, Gibbs RA, et al. (2010) Integrating common and rare genetic variation in diverse human populations. Nature 467: 52-58.

20. Purcell S, Neale B, Todd-Brown K, Thomas L, Ferreira MA, et al.(2007) PLINK: a tool set for whole-genome association and population-based linkage analyses. Am J Hum Genet 81: 559-575.

21. R development Core Team (2008) R: A language and environment for statistical computing. Vienna, Austria.

22. Peralta V, Cuesta MJ (2001) How many and which are the psychopathological dimensions in schizophrenia? Issues influencing their ascertainment. Schizophr Res 49: 269-285. 